\title{
Research productivity in animal learning from 1953 to 2000
}

\author{
MICHAEL DOMJAN and MARK A. KRAUSE \\ University of Texas, Austin, Texas
}

\begin{abstract}
Searches conducted with Medline and PsycInfo showed that the number of publications dealing with learning in animals increased between 1975 and 2000 and that the increase was substantially greater in Medline than in PsycInfo. An examination of major journals dealing with behavioral studies of conditioning and learning for the years 1953, 1963, 1973, 1983, 1993, and 2000 revealed a different pattern of results. The number of papers published in these journals increased from 1953 to 1973 but has been declining steadily since then. However, this decline was partially offset by an increase in the number of experiments published in each paper. Substantially more experiments were published in 2000 than in 1963 or 1953 . The number of core authors in the field also peaked in 1973 and has been declining since. However, there were only seven fewer core authors in 2000 than in 1983, and 1983 had as many core authors as 1963. These data suggest that pessimism about the status of behavioral studies of learning is not warranted if research activity is considered over a 40-year period. Furthermore, increased interest in the neural and biological mechanisms of learning should bode well for the status of behavioral research, because one cannot examine the physiological mechanisms of a behavioral process without first clearly understanding the phenomenon at the behavioral level.
\end{abstract}

Current research in animal learning ${ }^{1}$ does not enjoy the central role in experimental psychology that it had during the 1950s, when many of the leading learning theorists of the 20th century were still active professionally (e.g., Guthrie, Harlow, Mowrer, Spence, Skinner, and Tolman).

A variety of factors, some practical and some intellectual, have contributed to the perceived decline in animal learning research. Animal rights activists have made it more challenging to maintain animal laboratories (Turville-Heitz, 2000). The costs of doing animal research have increased substantially because of increased regulation. Grant support for research on basic behavioral mechanisms of learning seems difficult to obtain. Fewer graduate students seem to be interested in animal research, and most of the few students who are seem more interested in various aspects of the biological mechanisms of learning than in behavioral mechanisms. Jobs specifically calling for expertise in behavioral mechanisms of conditioning and learning seem to be few and far between. Also, experimental psychologists who were trained during the "cognitive revolution" in psychology have had little interest in animal learning.

Investigators of animal learning frequently discuss among themselves the various reasons for the perceived decline of the field. However, these discussions are spec-

We are grateful to Olivia Ortega, Mindy Fisher, and Leah Sanchez for their assistance in collecting the data presented in this paper. Correspondence should be addressed to M. Domjan, Department of Psychology, University of Texas at Austin, Austin, TX 78712 (e-mail: domjan@psyvax.psy.utexas.edu). ulative, because concrete data on the extent of research activity in animal learning is not available. The present report is intended to provide such information.

\section{Frequency of Publications in Medline and PsycInfo}

Medline provides broad coverage of the scientific literature in the biological and medical sciences, and PsycInfo provides broad coverage of the literature in psychology. To obtain a rough indication of the changes in research productivity in animal learning during the past 30 years, we conducted searches of Medline and PsycInfo, using the Ovid computerized search system. We entered learning as a search term and then limited the search to studies involving animals. We obtained numbers of papers in each database in 5-year blocks, starting in 1970.

The results are presented in Figure 1. Medline showed a steady increase in the numbers of publications dealing with learning limited to animals from 1970 to 1999. PsycInfo showed a modest decrease from 1970 to 1975 but a steady increase after that. Although the increase in PsycInfo was not as great as that in Medline, there were about 1,000 more publications dealing with learning limited to animals in 1995-1999 than in 1970-1974.

Figure 2 shows the proportion of all papers dealing with learning that involved animals in both databases. As would be expected, articles listed in Medline were more likely to involve animal subjects than were articles listed in PsycInfo. However, the proportion of papers dealing with learning that involved animals has been fairly steady since 1975 in both Medline and PsycInfo. 


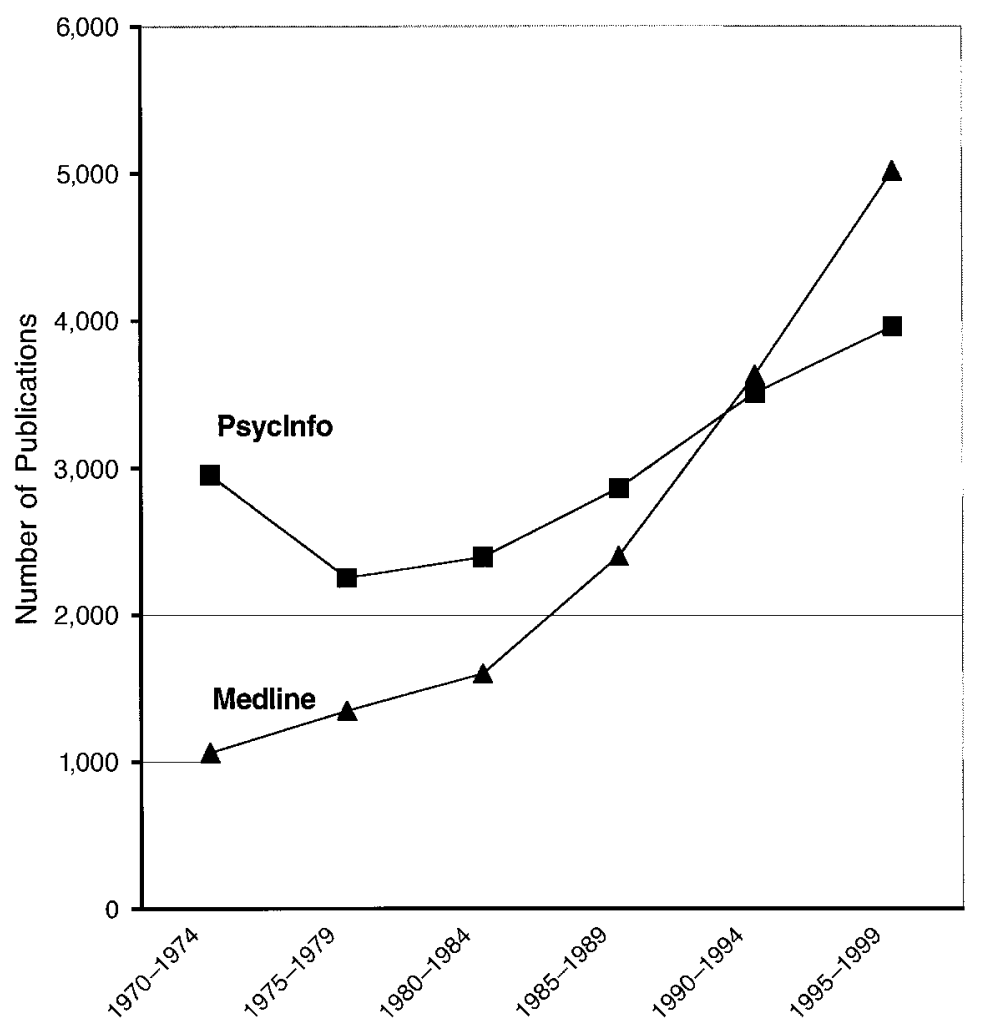

Figure 1. Frequency of publications in PsycInfo and Medline obtained by using learning as a search term and then limiting the search to studies involving animals in 5-year blocks from 1970 to 1999.

\section{Detailed Examination of Behavioral Publications in Animal Learning}

Although PsycInfo deals with the psychological rather than the biological literature, there is some overlap in the journals that are included in these two databases. PsycInfo does not exclude biological investigations. In the second part of our study, we examined patterns of research productivity limited to journals that publish primarily behavioral experiments. In addition, we obtained data not only on the frequency of publications, but also on other parameters of research productivity. We counted the number of papers that were published, the number of experiments that were reported, the number of animals that served in the experiments, the number of authors that contributed papers, and the number of active core authors (defined as two or more publications per year). Data were obtained for journals published in 1953, 1963, 1973, 1983, 1993, and 2000.

In 1953, only two prominent journals published studies of animal learning in the United States: the Journal of Experimental Psychology (JEP) and the Journal of Comparative and Physiological Psychology (JCPP). In 1963, prominent American journals for research in animal learning included JEP, JCPP, and the Journal of the Experimental Analysis of Behavior (JEAB). JEP, JCPP, and JEAB continued publication in 1973, and two additional prominent American journals for research in ani- mal learning became available: Learning and Motivation (L\&M) and Animal Learning \& Behavior (AL\&B). In 1983 and 1993, the prominent American journals for research in animal learning were JEAB, L\&M, AL\&B, and the Journal of Experimental Psychology: Animal Behavior Processes (JEP:ABP), one of the successors to JEP. In addition to the prominent American journals, for each year examined, we also included data from the Quarterly Journal of Experimental Psychology, the primary European journal for studies of animal learning.

The results are summarized in Table 1 . The rate of publication in animal learning was fairly low in 1953, with only 76 papers published. There was a steady increase in publications from 1953 to 1973 , when the greatest number of papers (256) was published in the major journals. The number of papers declined in each of the subsequent decades, with a total of only 114 papers published in 2000. However, this decline was partially offset by a steady increase in the number of experiments published per manuscript, from 1.03 in 1963 to 2.44 in 2000 . The total number of published papers decreased $55 \%$ in 2000 , relative to 1973 . However, the total number of experiments published decreased only $8.5 \%$ in 2000, relative to 1973 .

The year in which the greatest number of papers was published (1973) also saw the greatest number of subjects serving in the experiments $(9,126)$. Subject utiliza- 


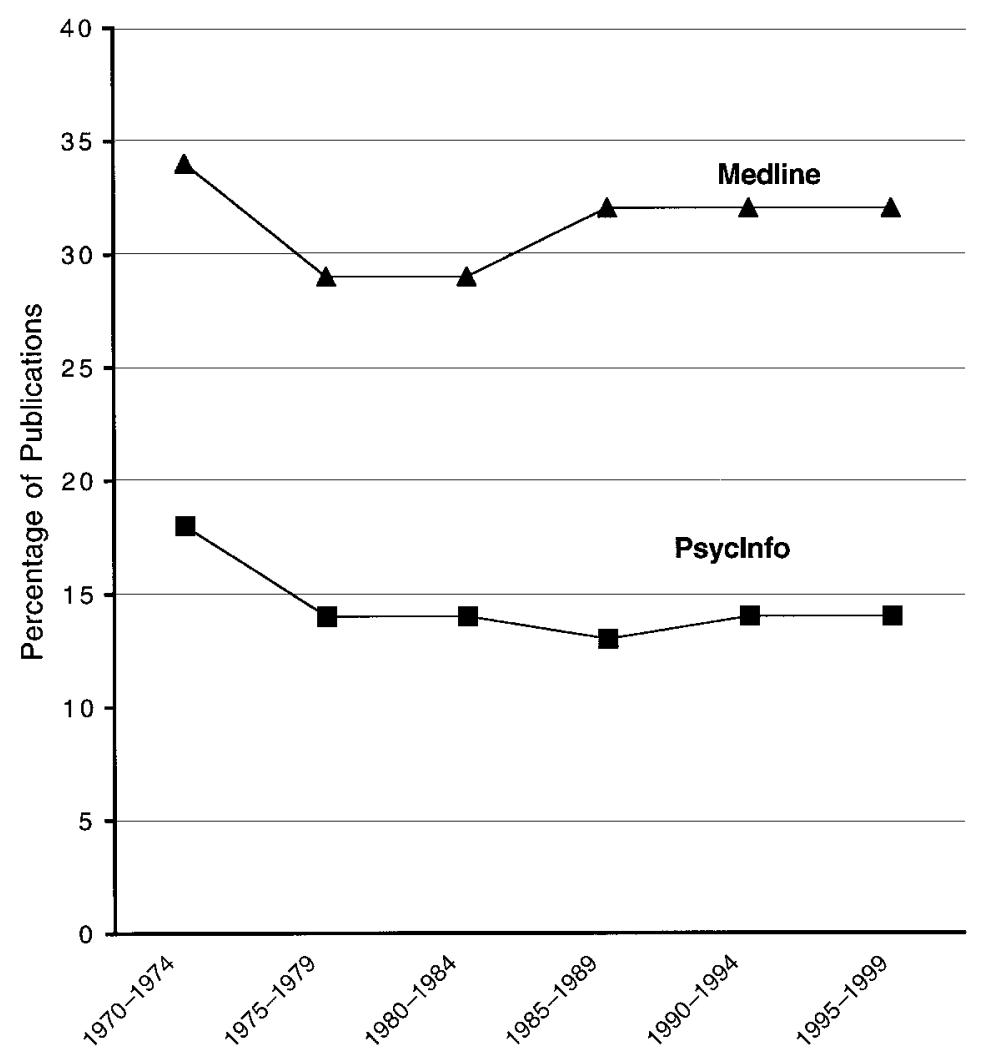

Figure 2. Percentages of all papers in learning that involved animals in PsycInfo and Medline in 5-year blocks from 1970 to 1999.

tion steadily declined after that, with only 6,381 subjects tested in 2000. This decrease in the total number of animals tested seems to have occurred because of increased efficiencies in the utilization of animal subjects. An average of 36.1 subjects served in each experiment in 1953. This number dramatically decreased (to $20.8 \mathrm{sub}-$ jects/experiment) from 1953 to 1983 and has increased only slightly (to 22.9 in 2000) since then.

How has the number of individuals engaged in research on animal learning changed since 1953 ? This question can be answered by examining the number of authors of papers in animal learning. As was the case with the number of papers published, the total number of authors increased from 1953 (123) to 1973 (475) and declined thereafter. The totals for the number of authors includes redundant entries, since many authors published more than a single paper during the years sampled. A pattern similar to that in the data on the total number of authors is evident when examining the total number of different authors in each decade. Despite the decline since 1973, the number of different authors was much higher in 2000 (240) than it was in 1953 (106) and

Table 1

Research Activity in Animal Learning

\begin{tabular}{lcccccc}
\hline \multicolumn{1}{c}{ Parameter } & 1953 & 1963 & 1973 & 1983 & 1993 & 2000 \\
\hline Number of papers & 76 & 172 & 256 & 179 & 147 & 114 \\
Number of experiments & 80 & 178 & 305 & 387 & 312 & 279 \\
Experiments per paper & 1.05 & 1.03 & 1.19 & 2.16 & 2.18 & 2.44 \\
Number of subjects & 2,885 & 6,346 & 9,126 & 8,039 & 6,785 & 6,381 \\
Subjects per experiment & 36.1 & 35.7 & 29.9 & 20.8 & 21.7 & 22.9 \\
Total number of authors & 123 & 323 & 475 & 402 & 333 & 291 \\
Number of different authors & 106 & 242 & 402 & 342 & 264 & 240 \\
Number of core authors* & 12 & 46 & 54 & 46 & 44 & 39 \\
Mean number of authors per paper & 1.62 & 1.88 & 1.86 & 2.25 & 2.27 & 2.55 \\
Mean number of publications per author & 1.39 & 1.41 & 1.57 & 1.91 & 1.80 & 2.11 \\
Mean number of publications per core author & 2.42 & 2.76 & 2.39 & 2.30 & 2.57 & 2.31 \\
\hline
\end{tabular}

*Core authors were designated as those who had published two or more articles during the sampled year. 
is comparable to the number of different authors in 1963 (242).

Another way to measure the size of the research community is to count the number of core authors. We defined someone as a core author if that person published two or more papers in a given year. As with other indexes of research activity, the number of core authors peaked in 1973 (54) and has been declining since. However, despite this decline, there were 39 core authors in 2000 , whereas there were only 12 core authors in 1953. In addition, the productivity of the core authors has remained remarkably stable. The mean number of publications per core author was 2.42 in 1953 and 2.31 in 2000.

\section{Discussion}

The results are noteworthy for several reasons. The data refute the contention that animal learning is becoming an extinct field. Our keyword searches in the PsycInfo and Medline databases showed either steady or increasing frequencies of papers dealing with animal learning topics between 1970 and 2000. This is hardly the pattern of results one would expect for a field that is dying out. These data also suggest that federal regulations, increased costs, and increased pressure from animal rights activists have not discouraged research in animal learning. The increasing number of papers on animal learning found in Medline was particularly striking and, no doubt, reflects an increasing number of researchers examining learning from neuroscience and biomedical perspectives.

In contrast to the results obtained from PsycInfo and Medline, detailed analyses of the data from the leading animal learning journals from 1953 to 2000 indicated that research activity has shown modest declines since 1973. However, research activity in 2000 , as measured in terms of the total number of experiments published, was still substantially higher than it was in 1953 or 1963. Thus, pessimism about the status of behavioral studies of learning is not warranted if research activity is considered over a longer period of time.

The increase in studies of learning in Medline versus the modest decline in the major behavioral journals in recent decades is also not grounds for much pessimism. These trends are bound to be reconciled in future patterns of research activity. Investigations of the physiological mechanisms of learning have to rest on a sound behavioral foundation. One cannot examine the physiological mechanisms of a behavioral process without first clearly understanding the phenomenon at the behavioral level. Rather than foretelling the further erosion of behavioral studies of learning, the growing interest in the neural and biological mechanisms of learning should underscore the continued need for sound behavioral analysis.

\section{REFERENCES}

Turville-Heitz, M. (2000, February). Violent opposition. Scientific American, 282, 32.

\section{NOTE}

1. The animal kingdom includes both human and nonhuman species. However, in discussions of animal research, the term animal has been used typically only in reference to nonhuman species. That usage is followed in the present paper.

(Manuscript received November 1, 2001; revision accepted for publication April 29, 2002.)

\section{Call for Papers \\ Special Issue on Pavlovian Conditioning and Basic Associative Processes}

The InternationalJournal of Comparative Psychology (edited by Mauricio Papini) is inviting manuscripts for a special issue entitled Pavlovian Conditioning and Basic Associative Processes, to be published in 2004. This issue will be devoted to studies examining the associative structures and processes of behavior in mammals and birds. Empirical studies are encouraged, although theoretical papers and review articles will be considered. Manuscripts should be approximately 20-30 pages long, not including figures, references, tables. The deadline for submission of manuscripts is March 31,2003. Three copies of manuscripts should be sent to the Guest Editor of this special issue: Todd R. Schachtman, Department of PsychologicalSciences, 210 McAlester Hall, University of Missouri, Columbia, MO 65211 (e-mail: schachtmant@ missouri.edu). 